\title{
Pericyte-specific expression of PDGF beta receptor in mouse models with normal and deficient PDGF beta receptor signaling
}

\author{
Ethan A Winkler ${ }^{\dagger}$, Robert D Bell ${ }^{\dagger}$, Berislav V Zlokovic $^{*}$
}

\begin{abstract}
Background: Pericytes are integral members of the neurovascular unit. Using mouse models lacking endothelialsecreted platelet derived growth factor-B (PDGF-B) or platelet derived growth factor receptor beta (PDGFRß) on pericytes, it has been demonstrated that PDGF-B/PDGFR $\beta$ interactions mediate pericyte recruitment to the vessel wall in the embryonic brain regulating the development of the cerebral microcirculation and the blood-brain barrier (BBB). Relatively little is known, however, about the roles of PDGF-B/PDGFR $\beta$ interactions and pericytes in the adult brain in part due to a lack of adequate and/or properly characterized experimental models. To address whether genetic disruption of PDGFR $\beta$ signaling would result in a pericyte-specific insult in adult mice, we studied the pattern and cellular distribution of PDGFR $\beta$ expression in the brain in adult control mice and F7 mice that express two hypomorphic Pdgfr $\beta$ alleles containing seven point mutations in the cytoplasmic domain of PDGFR $\beta$ that impair downstream PDGFR $\beta$ receptor signaling.

Results: Using dual fluorescent in situ hybridization, immunofluorescent staining for different cell types in the neurovascular unit, and a fluorescent in situ proximity ligation assay to visualize molecular PDGF-B/PDGFR $\beta$ interactions on brain tissue sections, we show for the first time that PDGFR $\beta$ is exclusively expressed in pericytes, and not in neurons, astrocytes or endothelial cells, in the adult brain of control 129S1/SvImJ mice. PDGFR $\beta$ colocalized only with well-established pericyte markers such as Chondroitin Sulfate Proteoglycan NG2 and the xLacZ4 transgenic reporter. We next confirm pericyte-specific PDGFR $\beta$ expression in the brains of F7 mutants and show that these mice are viable in spite of substantial $40-60 \%$ reductions in regional pericyte coverage of brain capillaries.

Conclusions: Our data show that PDGFR $\beta$ is exclusively expressed in pericytes in the adult 129S1/Sv1mJ and F7 mouse brain. Moreover, our findings suggest that genetic disruption of PDGFR $\beta$ signaling results in a pericytespecific insult in adult F7 mutants and will not exert a primary effect on neurons because PDGFR $\beta$ is not expressed in neurons of the adult 129S1/SvlmJ and F7 mouse brain. Therefore, mouse models with normal and deficient PDGFRß signaling on a 129S1/SvImJ background may effectively be used to deduce the specific roles of pericytes in maintaining the cerebral microcirculation and BBB integrity in the adult and aging brain as well as during neurodegenerative and brain vascular disorders.
\end{abstract}

\section{Background}

Pericytes are vascular mural cells embedded within the basement membrane of capillaries originally discovered by Rouget in 1873 [1]. In the central nervous system (CNS), pericytes are widely believed to be integral,

\footnotetext{
*Correspondence: berislav_zlokovic@urmc.rochester.edu † Contributed equally

Center for Neurodegenerative and Vascular Brain Disorders, Department of Neurosurgery, University of Rochester, Rochester, NY, USA
}

multifunctional members of the neurovascular unit at the capillary level [2-5]. Pericytes are seen to ensheathe microvascular endothelial cells forming multiple synapse-like "peg-socket" contacts with adjacent endothelial cells in brain capillaries suggesting the possibility of tightly regulated signaling and functional coupling between these two cell-types [4,6,7]. Although it has been known that brain capillaries have much greater pericyte coverage than peripheral vascular beds, the

\section{() Biomed Central}


presence and functional responsibilities of CNS pericytes have largely been neglected until the past two decades $[6,7]$.

Much of the recently gained insight into pericyte biology arose from the analysis of pericyte deficient transgenic mice with disrupted platelet derived growth factor $\mathrm{B}$ (PDGF-B)/platelet derived growth factor receptor beta (PDGFR $\beta$ ) signaling [8-13]. During development of brain capillaries PDGFR $\beta$ is exclusively expressed in perivascular pericytes $[10,14]$. In the embryonic neural tube, endothelial-secreted PDGF-B binds to the PDGFR $\beta$ receptor located on the pericyte plasma membrane resulting in dimerization of PDGFR $\beta$, subsequent autophosphorylation of cytoplasmic tyrosine residues and binding of $\mathrm{SH} 2$ domain containing proteins which in turn initiate a multitude of signal transduction pathways ultimately stimulating the proliferation, migration, and recruitment of pericytes to the vascular wall of newly formed blood vessels $[10,14,15]$. Complete knockout of $P d g f b$ or $P d g f r \beta$ results in a complete lack of pericytes and embryonic lethality [8,9]. Normal PDGF-B/ PDGFR $\beta$ interactions and corresponding pericyte recruitment have been demonstrated to play a pivotal role in the regulation of the cerebral microcirculation, including regulating angiogenesis, vascular stability, and integrity of the blood-brain barrier during embryonic development $[2,7,16]$. Although it has been speculated that brain pericytes might fulfill similar roles in the adult brain, there is limited in vivo experimental evidence to support such claims. Therefore, the functional roles of brain pericytes in the adult and aging brain are relatively less well understood in part due to a lack of adequate and/or properly characterized experimental models.

To address whether genetic disruption of PDGFR $\beta$ signaling would result in a pericyte-specific insult in adult mice and may therefore be used to study the roles of brain pericytes in the adult and aging brain, we sought to characterize the pattern of expression of PDGFR $\beta$ in the adult mouse brain in both control mice and viable F7 mice with two hypomorphic Pdgfr $\beta$ alleles on a $129 \mathrm{~S} 1 / \mathrm{Svlm}$ J genetic background [12]. The F7 mice were generated by seven point mutations in which multiple cytoplasmic tyrosine residues at positions 578,715 , $739,750,770$ and 1008 were substituted with phenylalanine and the tyrosine at position 1020 was replaced with isoleucine. As a result of these mutations, Src-, Grb2-,

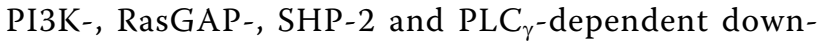
stream signaling of the PDGFR $\beta$ receptor is disrupted. Impaired PDGFR $\beta$ downstream signaling in turn results in diminished pericyte recruitment to the vessel wall leading to a $55-75 \%$ reduction in the number of pericytes in the embryonic CNS at day E14.5 as previously shown [12]. However, the effects of such alterations in
PDGFR $\beta$ signaling in the adult mouse brain remain unknown.

Through dual fluorescent in situ hybridization (FISH), triple immunofluorescent staining for different cell types in the neurovascular unit, and a fluorescent in situ proximity ligation assay (PLA) to visualize molecular PDGF-B/PDGFR $\beta$ interactions on brain tissue sections, we show for the first time that PDGFR $\beta$ is exclusively expressed in pericytes in several brain regions in the adult control 129S1/SvlmJ mouse model, and confirm a similar pattern of pericyte-specific PDGFR $\beta$ expression in the adult brain in F7 mutants on the same genetic background. We demonstrate that F7 mutants are viable, but exhibit substantial pericyte reductions in different brain regions offering a prospective model system appropriate for examining the role of pericytes in the adult and aging brain.

\section{Results}

PDGFR $\beta$ pericyte-specific expression in the adult brain of 129S1/SvlmJ mice and F7 mutants

The adult murine cerebrum is comprised of multiple distinct brain structures, including the cortex and hippocampus, each demonstrating a unique cytoarchitecture. In order to provide orientation for later fluorescent images, we first utilized hematoxylin and eosin histological staining to provide a low magnification view of an adult control 129S1/SvlmJ mouse cerebral hemisphere adjacent to sections subsequently utilized for fluorescent imaging experiments (Figure 1a, panel i). Further higher magnification analysis demonstrates the distinct layered cytoarchitecture and close approximation between vascular cells, neurons, and glia in the murine parietal cortex (Figure 1a, panel ii) and CA1 subfield of the hippocampus (Figure 1a, panel iii).

We then determined the expression pattern of $P d g f r \beta$ mRNA in adult control 129S1/SvlmJ 6 month old mouse cortex and hippocampus. We show that Pdgfr $\beta$ mRNA is exclusively expressed in perivascular cells at the capillary level as demonstrated in layer III of the parietal cortex and CA1 subfield of the hippocampus (Figure $1 \mathrm{~b}$, left and right panels), by performing dual fluorescent in situ hybridization combined with endothelial-specific GLUT1 immunostaining. Pdgfr $\beta$ mRNA expression was restricted to perivascular cells adjacent to GLUT1-positive brain capillaries in frontal and temporal cortices as well as the striatum in the adult 129S1/SvlmJ mouse brain (data not shown).

Next, we studied whether PDGFR $\beta$ is an abundant marker expressed in brain pericytes at the protein level that would enable us to quantify pericyte coverage in various brain regions. We show that PDGFR $\beta$ is highly expressed in pericytes on lectin-positive brain capillaries as illustrated in both layer III of the parietal cortex and 

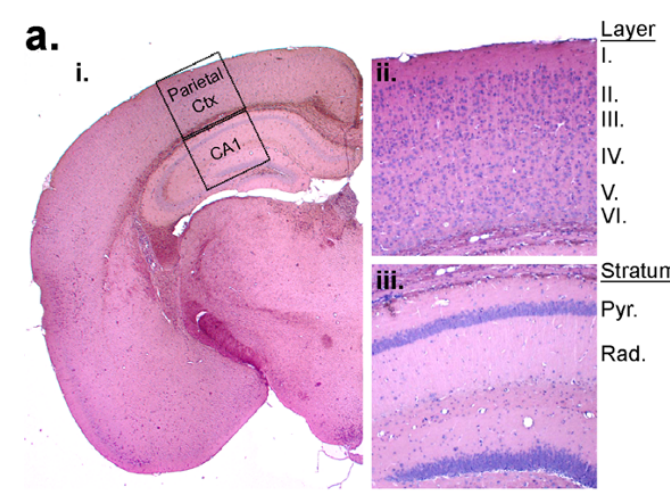

CA1

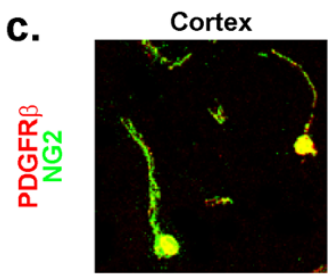

Hippocampus
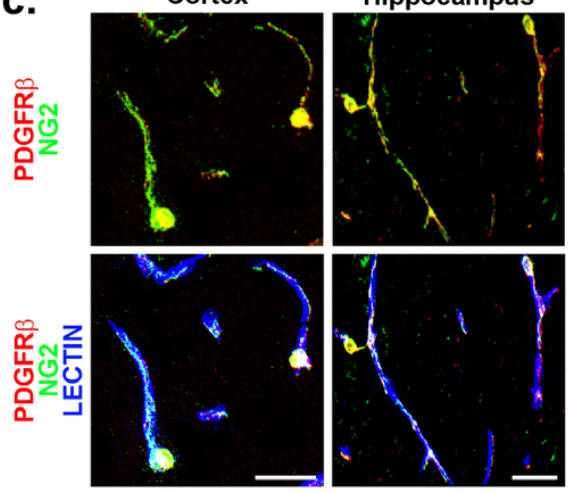

e.

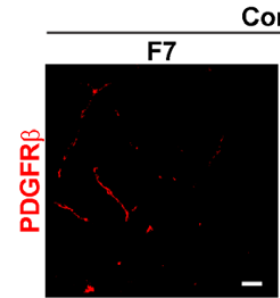

Control
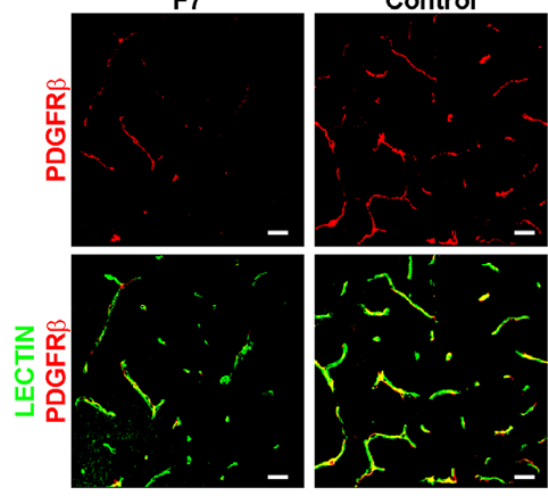

b.
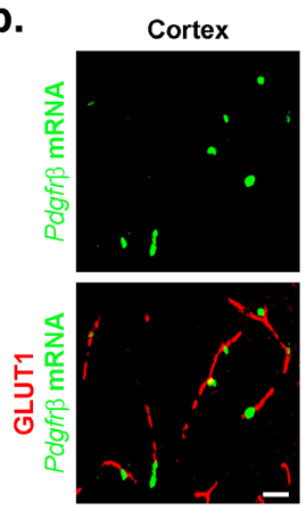

CA1

Hippocampus
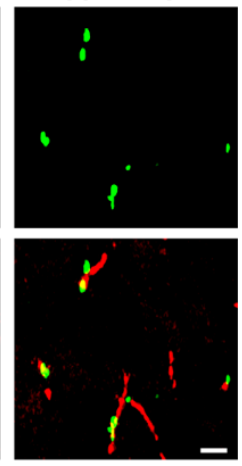

d.

Cortex

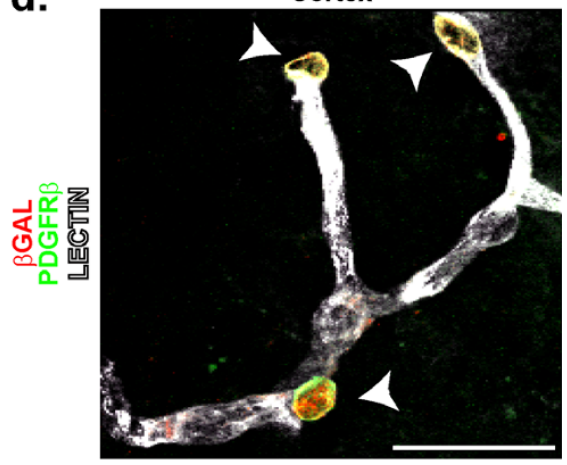

f.

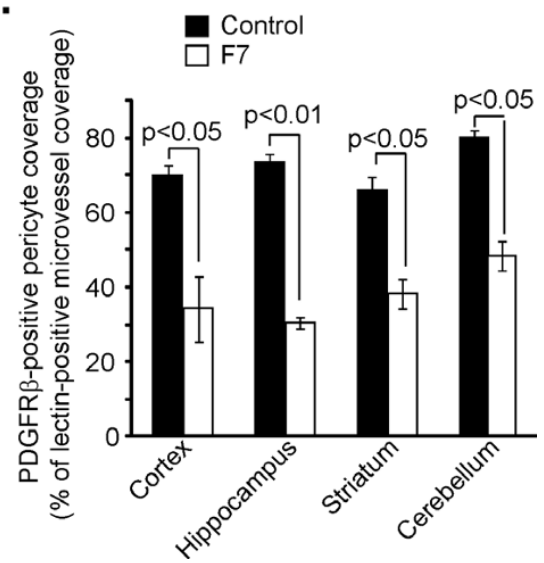

Figure 1 PDGFR $\beta$ expression in perivascular pericytes in the adult brain in control 129S1/SvlmJ mice and F7 mutants with two hypomorphic PDGFR $\boldsymbol{\beta}$ alleles. (a) Bright-field imaging analysis of hematoxylin and eosin stained 6 month old 129S1/SvlmJ mouse cerebral hemisphere $\mathbf{i}$. Low magnification image depicting brain regions used for fluorescent imaging experiments (broken rectangles). Parietal Ctx, Parietal Cortex; CA1, CA1 hippocampal subfield. ii-iii. Higher magnification image depicting the layered cytoarchitecture of the somatosensory parietal cortex (ii) and the CA1 hippocampal subfield (iii). Roman numerals represent cortical layers. Pyr., Pyramidale; Rad., Radiatum. (b) Representative confocal imaging analysis of dual in situ hybridization for Pdgfr $\boldsymbol{\beta}$ mRNA (green) and endothelial-specific GLUT1 immunodetection in brain capillaries (red) in layer III of the parietal cortex (left) and the stratum pyramidale of the CA1 hippocampal subfield (right) in 6 month old control 129S1/SvImJ mouse. Scale bar 20 um. (c) Representative three-color confocal imaging analysis of PDGFRß immunodetection (red), NG2 immunodetection of perivascular pericytes (green), and lectin-positive brain capillaries (blue) in layer III of the parietal cortex (left) and stratum pyramidale of the CA1 hippocampal subfield (right) in a 6 month old 129S1/SvlmJ mouse. Colocalization: yellow. Scale bar $20 \mu \mathrm{m}$. (d) Representative three-color confocal imaging analysis of PDGFR $\beta$ immunodetection (green), $\beta$-gal nuclear immunodetection of pericyte-specific LacZ transgene (red), and lectin-positive brain capillaries (white) in layer III of the parietal cortex in a 2 month old $x$ LacZ4 transgenic mouse. Colocalization, arrows: yellow. Scale bar $20 \mu \mathrm{m}$. (e) Representative confocal imaging analysis of PDGFR $\beta$ immunodetection (red) and lectinpositive brain capillaries (green) in the parietal cortex of a 6 month old 12951/SvImJ littermate control (right) and F7 mouse (left). (f) Graph: PDGFR $\beta$-positive pericyte coverage of lectin-positive brain capillary profiles in the cortex, hippocampus, striatum and cerebellum in 6-8 month old 129S1/SvImJ controls and F7 mice. Mean \pm SEM and $n=6$ animals per genotype. 
CA1 subfield of the hippocampus (Figure 1c, left and right panels) by performing three-color immunofluorescent staining for PDGFR $\beta$ and the well-established CNS pericyte marker NG2 [7,17-19] along with endothelialspecific Lycopersicon Esculentum lectin fluorescent staining to visualize brain capillaries. To further confirm pericyte-specific expression of PDGFR $\beta$, we utilized the $x$ LacZ4 transgenic mouse model which specifically expresses the lac $Z$ nuclear transgenic reporter in committed, differentiated and non-proliferating pericytes/ vascular smooth muscle cells [20]. Double immunofluorescent staining demonstrates that PDGFR $\beta$ is exclusively expressed in pericytes with $\beta$-galactosidase $(\beta$-gal) positive nuclear staining located on lectin-positive brain capillaries in both layer III of the parietal cortex (Figure $1 \mathrm{~d}$ ) and CA1 subfield of the hippocampus (data not shown). PDGFR $\beta$ expression was also restricted to NG2or nuclear $\beta$-gal-positive pericytes located on lectinpositive brain capillaries in frontal and temporal cortices as well as the striatum in the adult $129 \mathrm{~S} 1 / \mathrm{Svlm}$ J mouse brain (data not shown).

As shown in Figure 1c-e, PDGFR $\beta$ immunofluorescent staining provides a quantitative fluorescent signal that may be used to determine pericyte coverage of microvascular profiles in mouse brain tissue. Quantification of pericyte coverage in the adult 6-8 month old control 129S1/SvlmJ mouse cortex, hippocampus, striatum and cerebellum revealed a 70,73, 66, and $80 \%$ PDGFR $\beta$ positive pericyte coverage of lectin-positive microvessels, respectively (Figure 1f). Similar analysis of 6-8 month old F7 mutant mice revealed a $34,30,38,48 \%$ PDGFR $\beta$-positive pericyte coverage in the cortex, hippocampus, striatum and cerebellum, respectively (Figure $1 \mathrm{e}-\mathrm{f})$, indicating a substantial pericyte reduction by $52 \%$, $59 \%, 43 \%$, and $40 \%$, respectively, compared to controls in different brain regions in this mouse model. The observed reductions in pericyte coverage in the F7 mice were subsequently confirmed in the cortex and hippocampus using desmin, another well-established pericyte marker [14,15,21,22]. Quantification of desmin-positive pericyte coverage in the adult 6-8 month old control 129S1/SvlmJ and F7 mouse cortex and hippocampus revealed a similar $52 \%$ and $59 \%$ reduction in pericyte coverage of lectin-positive microvessels, respectively.

\section{PDGFR $\beta$ is not expressed in neurons or astrocytes in the adult brain of $129 \mathrm{~S} 1 / \mathrm{SvlmJ}$ mice and F7 mutants}

We failed to detect any mouse Pdgfr $\beta$ mRNA by in situ hybridization or PDGFR $\beta$ immunostaining by high resolution confocal microscopy in any other cell types in the adult control 129S1/SvlmJ or F7 mouse brain (Figure 1be; Figure 2). To confirm that there is no PDGFR $\beta$ expression in other cell types in the adult 129S1/SvlmJ and F7 mouse brain, we utilized double immunofluorescent detection coupled to high resolution confocal microscopy imaging of PDGFR $\beta$ and either the neurofilament- $\mathrm{H}$ marker of neuronal cell processes (SMI-32) (Figure 2a, b), the neuronal-specific nuclear antigen A60 (NeuN) (Figure 2c, d), or the astrocyte-specific glial fibrillary acidic protein (GFAP) (Figure 2e, f) along with endothelial-specific fluorescent lectin staining. We show no detectable colocalization of PDGFR $\beta$ immunofluorescent signal with any of the above cell-specific markers in cortex (Figure 2a, c, e), hippocampus (Figure 2b, d, f), or striatum (data not shown) in the adult 129S1/SvlmJ or F7 mouse brain.

In order to investigate whether a similar pericyterestricted pattern of PDGFR $\beta$ expression is also present in non-telencephalic brain structures, we conducted double immunofluorescent detection experiments in the dorsal medulla oblongata including the nucleus tractus solitarius, a nucleus previously reported to have neuronal PDGFR $\beta$ expression [23,24]. In contrast to these published results, there was no detectable colocalization of PDGFR $\beta$ immunofluorescent signal with NeuN-positive neurons (Figure 2g) or GFAP-positive astrocytes (data not shown).

\section{PDGF-B/PDGFR $\beta$ interactions occur only on perivascular pericytes in the adult mouse brain on 129S1/SvImJ genetic background}

To further confirm that PDGFR $\beta$ is only expressed on brain pericytes and that a lack of PDGFR $\beta$ on other CNS cell types is not due to insufficient sensitivity of conventional immunofluorescent staining, we designed a fluorescent in situ proximity ligation assay (PLA) to visualize molecular PDGF-B/PDGFR $\beta$ interactions in the adult $129 \mathrm{~S} 1 / \mathrm{Svlm}$ J mouse brain. PLA is a well-established, antibody based technique designed to visualize endogenous protein-protein interactions with sufficient amplification to detect single molecular events without requiring protein modification, e.g., the creation of fluorescently tagged fusion proteins $[25,26]$. We show that PDGF-B/PDGFR $\beta$ interactions occur only on PDGFR $\beta$-positive pericytes located on lectin-positive brain capillaries in layer III of the parietal cortex (Figure 3a), CA1 subfield of the hippocampus (Figure $3 \mathrm{~b}$ ), and striatum (data not shown) in the adult 129S1/SvlmJ mouse brain.

\section{Discussion}

As our results demonstrate, PDGFR $\beta$ is exclusively expressed in brain pericytes and not in neurons, astrocytes or endothelial cells in adult, viable mouse models with normal and deficient PDGFR $\beta$ signaling, such as the F7 mutants, on a 129S1/SvlmJ genetic background. Significantly, this pericyte-specific PDGFR $\beta$ expression is observed at both the mRNA and protein level in 
a.

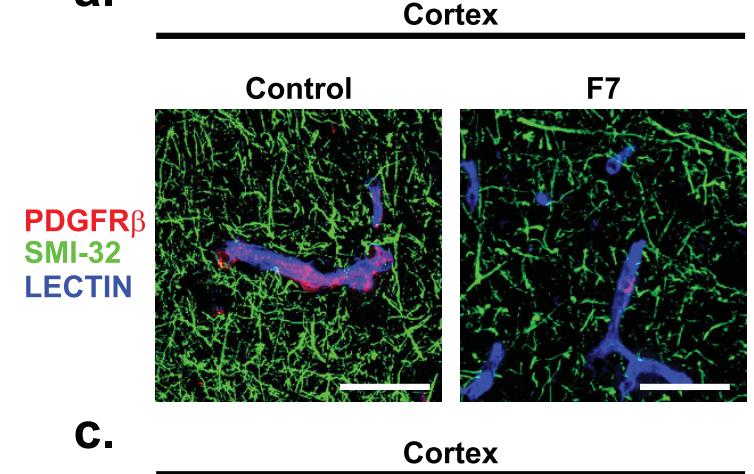

C.

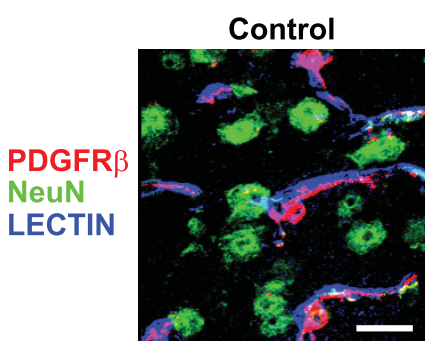

e.

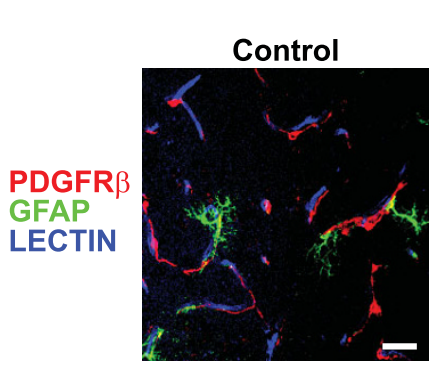

Cortex
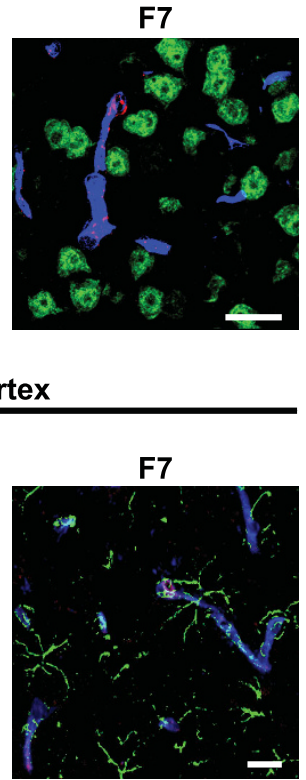

b.

\section{CA1 Hippocampus}
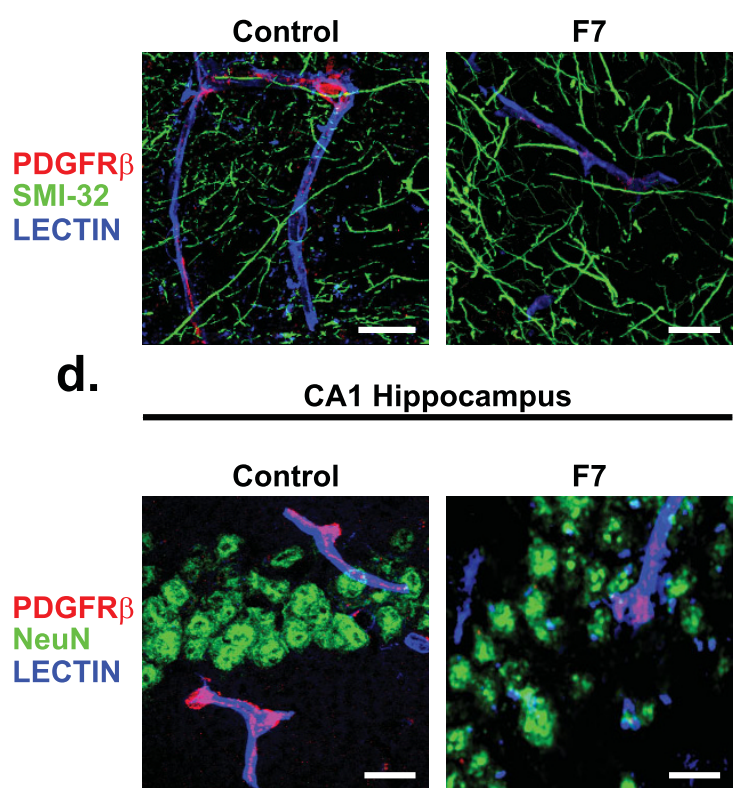

f.

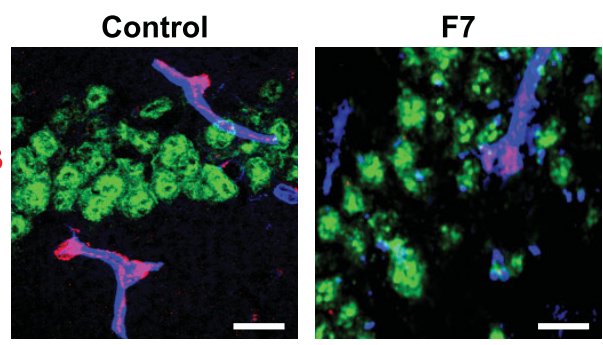

CA1 Hippocampus
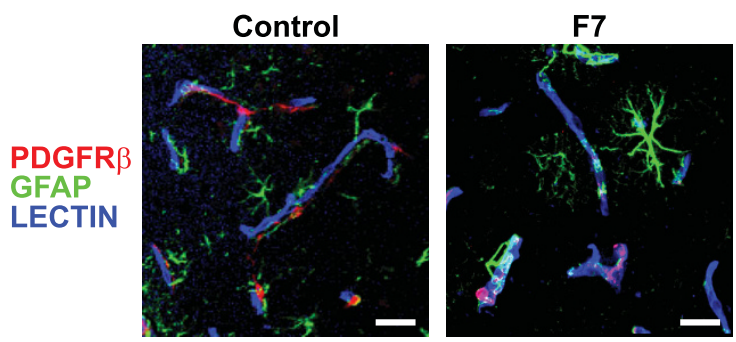

g. Nucleus Tractus Solitarius

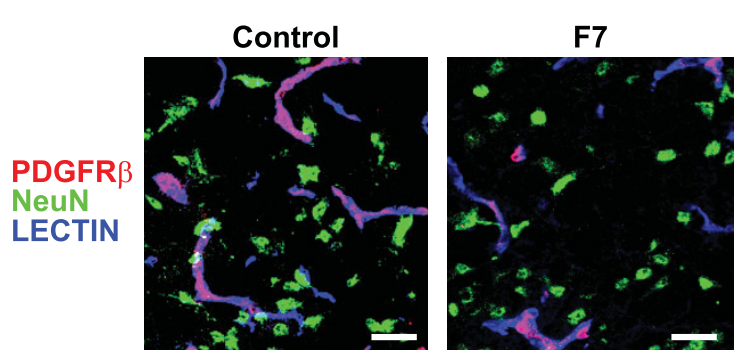

Figure 2 PDGFR $\beta$ is not expressed in neurons or astrocytes in the adult $129 \mathrm{~S} 1 / \mathrm{Svlm}$ J and F7 mouse brain. (a, b) Representative confocal imaging analysis of PDGFR $\beta$ immunodetection of perivascular pericytes (red), SMI-32 immunodetection of neuronal neurites (green), and lectinpositive brain capillaries (blue) in 6 month old 129S1/SvImJ control and 6 month old F7 mouse layer II parietal cortex (a) and stratum radiatum of the CA1 hippocampal subfield (b). Scale bar $20 \mu \mathrm{m}$. (c, d) Representative confocal imaging analysis of PDGFR $\beta$ immunodetection of perivascular pericytes (red), NeuN-specific immunodetection of neurons (green), and lectin-positive brain capillaries (blue) in 6 month old 129S1/ SvImJ control and 6 month old F7 mouse layer III parietal cortex (c) and stratum pyramidale of the CA1 hippocampal subfield (d). Scale bar 20 $\mu \mathrm{m}$. (e, f) Representative confocal imaging analysis of PDGFRß immunodetection of perivascular pericytes (red), GFAP-specific immunodetection of astrocytes (green), and lectin-positive brain capillaries (blue) in 6 month old 129S1/SvImJ control and 6 month old F7 mouse layer III parietal cortex (e) and stratum pyramidale of the CA1 hippocampal subfield (f). Scale bar $20 \mu \mathrm{m}$. (g) Representative confocal imaging analysis of PDGFR $\beta$ immunodetection of perivascular pericytes (red), NeuN-specific immunodetection of neurons (green), and lectin-positive brain capillaries (blue) in 6 month old 129S1/SvImJ control and F7 mouse nucleus tractus solitarius in dorsal medulla oblongata. Scale bar $20 \mu \mathrm{m}$. 


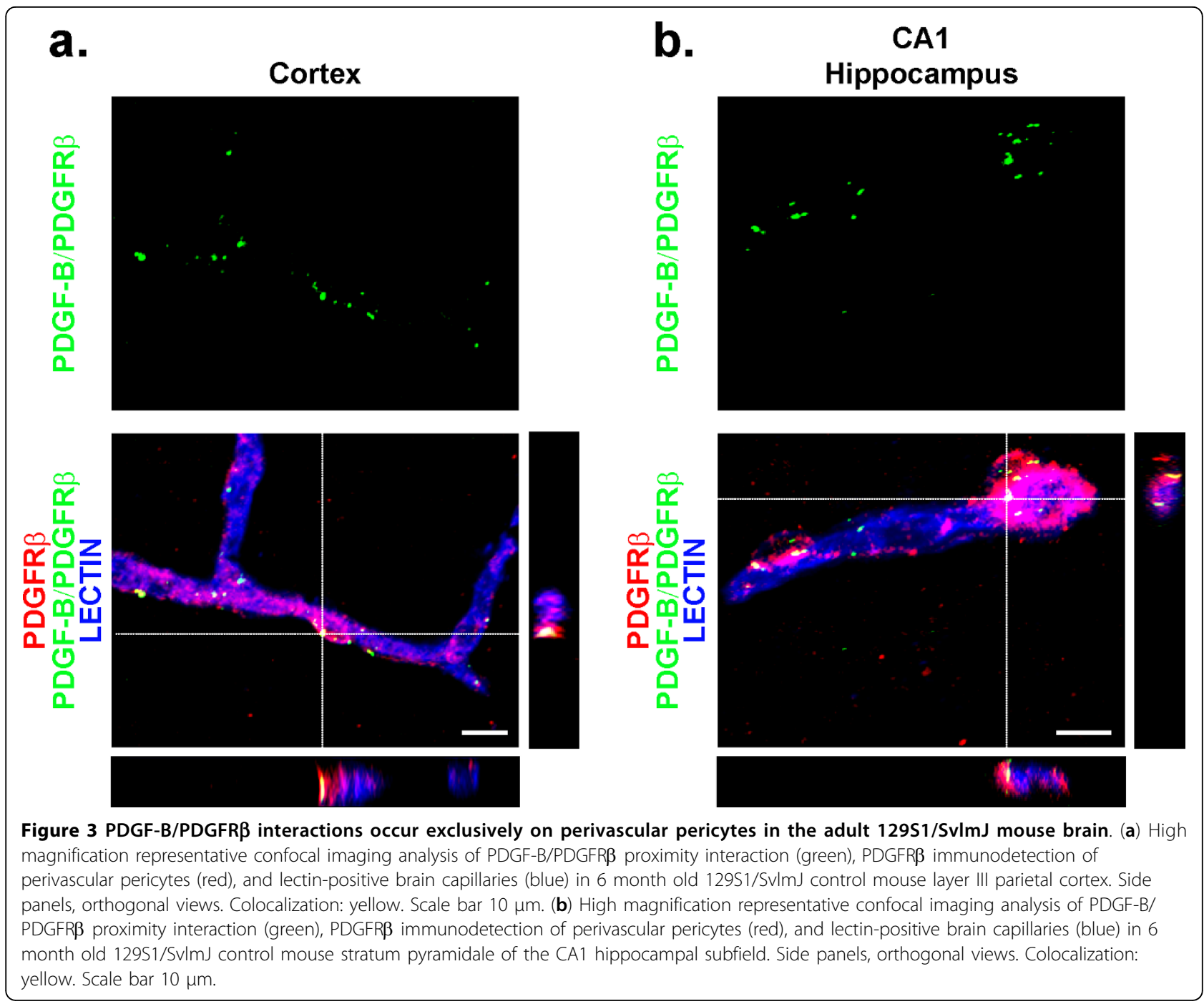

multiple brain regions. Furthermore, PDGFR $\beta$ expression is consistently restricted to brain pericytes with use of multiple fixatives and tissue processing techniques during immunofluorescent staining (see Methods). Previous studies utilizing mice on a 129Sv/C57BL6 genetic background have also found a similar pericyte-restricted pattern of PDGFR $\beta$ expression in mouse brain capillaries during embryonic development $[10,14]$. Moreover, gene expression profiling on acutely isolated neurons from C57BL6 mice and S100 $\beta$ (S100 calcium-binding protein $\mathrm{B}$ ) transgenic mice expressing enhanced green fluorescent protein on a hybrid C57BL6/DBA background failed to detect expression of $P d g f r \beta$ in neuronal cell populations $[27,28]$.

Intriguingly, recent in vitro studies utilizing primary cortical neurons isolated from C57BL6 mice during postnatal day 1 and 6 day old cultures have claimed PDGFR $\beta$ expression in cultured neurons after 6 days [29] in contrast to the previous report on acutely isolated non-cultured neurons from C57BL6 mice or mice on C57BL6/DBA genetic background showing a lack of Pdgfrb mRNA expression [27,28]. It remains unclear as to whether this discrepancy is the result of alterations in gene expression in response to the growth factors and/or other constituents in the medium during culturing [29], and may therefore not accurately represent the transcriptional profile of neuronal cell populations in vivo, as suggested by other studies [30-32] or after an acute isolation of neurons $[27,28]$.

Other studies utilizing adult C57BL6 mice have further claimed that PDGFR $\beta$ is expressed in mouse cortical neurons through ex vivo immunofluorescent staining and low resolution epi-fluorescent imaging $[23,33]$. Although these reports demonstrate PDGFR $\beta$ labeling consistent with neurons in size, shape and distribution, no colocalization studies were conducted and there is no concluding evidence against or in favor of neuronal PDGFR $\beta$ expression. In Ishii et al. 2006 [33], 
the authors further claim to have deleted PDGFR $\beta$ specifically from neurons utilizing a nestin-Cre transgenic system and demonstrate no neuronal phenotype but an exacerbated response to cryogenic injury of nestin-Cre

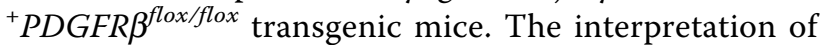
Ishii et al. experiments [33] is, however, complicated by recent findings demonstrating that brain pericytes express nestin [34] and therefore PDGFR $\beta$ expression in pericytes can be affected by nestin-Cre genetic manipulation. Since pericyte number and/or coverage have not been determined in the nestin-Cre $e^{+} P D G F R \beta^{\text {flox fllox }}$ transgenic mouse model [33], it remains uncertain to what extent the observed susceptibility to cryogenic brain injury in this mouse model is due to brain pericyte loss as opposed to primary neuronal insult.

The same group utilizing organotypic brainstem slices from mice on a C57BL6 background in conjunction with single cell patch clamp neuronal recordings has suggested that neuronal PDGFR $\beta$ mediates changes in the excitatory postsynaptic currents following application of human recombinant PDGF-BB onto the slices $[23,24]$. It is of note that PDGF-BB binds to other transmembrane receptors such as platelet derived growth factor receptor alpha (PDGFR $\alpha$ ) which is abundantly expressed on neurons in the murine CNS [35-39] and can contribute to the observed changes in postsynaptic currents. As a result, it remains unclear as to whether the observed effects are mediated by binding of recombinant PDGF-BB to neuronal PDGFR $\alpha$ at the concentration utilized and/or whether accompanying PDGF-B/ PDGFR $\beta$ signaling on brain pericytes may indeed influence neuronal electrophysiological responses further complicating the interpretation of these experiments [24]. In support of our argument, using an in situ proximity ligation assay capable of detecting single molecular events, we provide evidence demonstrating that detectable PDGF-B/PDGR $\beta$ interactions on tissue sections occurs exclusively on microvascular pericytes following exogenous application of equivalent concentrations (150 $\mathrm{ng} / \mathrm{mL}$ ) of recombinant murine PDGF-BB as in a previous study [24].

The source of the above contradictions between reports demonstrating a lack of neuronal PDGFR $\beta$ expression in vivo in mouse models on a 129S1/SvlmJ background in the present study and 129SV/C57BL6 background $[10,14]$ and reports suggesting the presence of neuronal PDGFR $\beta$ expression in mouse models on a C57BL6 background, including the nestin-Cre ${ }^{+}$PDGFR $\beta^{\text {flox } / \text { flox }}$ transgenic mouse model [23,24,33] remains unclear at present time. It is possible, however, that these conflicting results may be due to subtle genetic differences between mouse strains with different genetic backgrounds, as reported by others showing significant strain-specific responses to injury and strain- specific gene expression mapping in the adult mouse brain [40-43]. Intriguingly, previous reports have demonstrated neuronal PDGFR $\beta$ expression in the rat CNS [44], whereas our work in progress in the adult human CNS and Alzheimer's disease patients shows a pericyte-restricted pattern of PDGFR $\beta$ expression [Winkler EA, Bell RD, Zlokovic BV, unpublished results] indicating that species-specific differences in PDGFR $\beta$ expression may indeed exist and that the present mouse models on a 129S1/SvlmJ background could be potentially used as correlates for human studies on brain pericytes.

\section{Conclusions}

Here we have demonstrated that PDGFR $\beta$ is exclusively expressed in brain pericytes and may serve as an abundant pericyte-specific marker in the adult $129 \mathrm{~S} 1 / \mathrm{Sv} 1 \mathrm{~mJ}$ and F7 mouse brain. Moreover, these findings suggest that genetic disruption of PDGFR $\beta$ signaling in adult, viable mice with this genetic background, such as the F7 mice, will result in a pericyte-specific insult and will not exert a primary effect on neurons because PDGFR $\beta$ is not expressed in neurons of the adult 129S1/SvlmJ and F7 mouse brain. Therefore, mouse models with normal and deficient PDGFR $\beta$ signaling on a $129 \mathrm{~S} 1 / \mathrm{Svlm}$ J genetic background may effectively be used to deduce the specific roles of pericytes in maintaining the cerebral microcirculation and BBB integrity within the neurovascular unit in the adult and aging brain as well as during neurodegenerative and vascular brain disorders.

\section{Methods \\ Animals}

Transgenic F7 mutants and their littermate controls on a $129 \mathrm{~S} 1 / \mathrm{SvlmJ}$ genetic background were provided by Dr. Philippe Soriano (Fred Hutchinson Cancer Res. Cntr., Seattle, WA). The F7 mutants were generated by point mutations in which multiple cytoplasmic tyrosine residues at positions 578, 715, 739 and 750, 770, 1008 were substituted with phenylalanine and the tyrosine at position 1020 was replaced with isoleucine, thereby disrupting Src-, Grb2-, PI3K-, RasGAP-, SHP-2 and PLC $\gamma^{-}$ dependent signal transduction, respectively, as previously described [12]. This results in a substantial pericyte loss in the embryonic CNS, as shown at day E14.5 [12]. xLacZ4 mice were provided by Dr. Michelle Tallquist (UT Southwestern, Dallas, TX). Mice were cared for in accordance to the University of Rochester Medical Center Vivarium and Division of Laboratory Animal Medicine guidelines. All procedures were approved by the Institutional Animal Care and Use Committee at the University of Rochester using National Institute of Health guidelines. Mice were anesthetized with a single intraperitoneal injection of ketamine $(100 \mathrm{mg} / \mathrm{kg})$ and 
xylazine $(10 \mathrm{mg} / \mathrm{kg})$ and then transcardially perfused with $50 \mathrm{~mL}$ PBS containing $5 \mathrm{U} / \mathrm{ml}$ heparin. Brain sections were prepared as described below.

\section{Hematoxylin and Eosin Histologic Staining}

This was performed to provide a detailed view of cerebral cytoarchitecture and orientation for fluorescent imaging. Mice were anesthetized, euthanized, and perfused as described above (see Animals). Tissue sections were subsequently fixed overnight in $4 \%$ paraformaldehyde (PFA.) Staining was conducted utilizing the FD hematoxylin solution and FD eosin Y solution (FD Neurotechnologies; Catonsville, MD) as described by the manufacturer.

\section{Digoxigenein (DIG) labeled oligonucleotides}

A $129 \mathrm{~S} 1 / \mathrm{Svlm}$ J mouse brain cDNA library was created from freshly dissected mouse cortex. A $555 \mathrm{bp}$ fragment of $P d g f r \beta$ cDNA was selectively amplified using the following PCR primer combination: 5'- ACCTAGTCGACCACCTTTGTTCTG ACCTGCTC-3' and 5'TTCGTGGATCCATGGTGATGCTCTCGCCCT-3'. To facilitate directional cloning, primers were designed to include a $5^{\prime}$ overhang containing a 5 nucleotide spacer sequence and either a Sall or BamHI restriction site in the forward and reverse primer, respectively. The resulting $P d g f r \beta$ amplicon was subsequently ligated into the pBLUESCRIPT II SK vector (Stratagene; Cedar Creek, $\mathrm{TX}$ ) containing both T3 and T7 RNA polymerase sequences. The plasmid was sent to ACGT Inc. (Wheeling, IL) for sequencing to verify proper insertion. The inserted sequence subsequently aligned to $\mathrm{Mus} \mathrm{Muscu-}$ lus Pdgfr $\beta$ mRNA. Both sense and antisense DIG-labeled riboprobes were synthesized with the DIG RNA labeling kit (Roche Applied Science; Indianapolis, IN) utilizing T7 and T3 RNA polymerase, respectively. The DIGlabeled riboprobes were then purified using G-50 sephadex quick spin columns (Roche Applied Science) and diluted to $100 \mathrm{ng} / \mu \mathrm{L}$ in THE RNA storage solution (Ambion; Austin, TX). All riboprobes were aliquoted and stored at $-80^{\circ} \mathrm{C}$.

\section{Dual fluorescent in situ hybridization}

The brains of 6-8 month old 129S1/SvlmJ control mice were studied to determine the pattern of $P \operatorname{dgfr} \beta$ mRNA expression in the adult brain utilizing dual fluorescent in situ hybridization and immunofluorescent staining as previously described $[45,46]$. Mice were anesthetized and transcardially perfused as described above (see Animals). Snap frozen brains were cryosectioned at $20 \mu \mathrm{m}$ and stored at $-80^{\circ} \mathrm{C}$. The following steps were performed at $25^{\circ} \mathrm{C}$ unless otherwise indicated. All solutions were made using nuclease-free water and glassware was precleaned using RNase Zap reagent (Ambion). In brief, sections were allowed to dry for 30 minutes and then immediately fixed with 4\% PFA for 20 minutes. Sections were then incubated with $0.1 \mathrm{M}$ triethanolamine containing $0.25 \%(\mathrm{v} / \mathrm{v})$ acetic anhydride to minimize nonspecific binding of the riboprobe for 10 minutes and were treated with $10 \mu \mathrm{g} / \mathrm{mL}$ proteinase $\mathrm{K}$ (Ambion) diluted in PBS for 10 minutes to facilitate tissue penetrance. Sections were then incubated in $5 \times$ SSC $(0.75 \mathrm{M}$ $\mathrm{NaCl}, 0.75 \mathrm{M} \mathrm{Na}$-citrate) for $15 \mathrm{~min}$ and then prehybridized with prehybridization buffer $(5 \times$ SSC, $50 \%$ formamide, $\mathrm{pH}$ to 7.5 with $\mathrm{HCl}, 100 \mu \mathrm{g} / \mathrm{ml}$ sheared fish sperm DNA) for 2 hours at $65^{\circ} \mathrm{C}$. Sections were placed in a humidified chamber and incubated with $300 \mathrm{ng} / \mathrm{ml}$ DIG-labeled probe diluted in prehybridization buffer for $12-16$ hours at $65^{\circ} \mathrm{C}$. Sections were hybridized with either antisense or sense riboprobe as a negative control. Following hybridization, slides were washed with $2 \times$ SSC and $0.1 \times$ SSC for 1 hour at $70^{\circ} \mathrm{C}$. Sections were rinsed with PBS and incubated in $3 \% \mathrm{H}_{2} \mathrm{O}_{2}$ for 1 hour to block endogenous peroxidase activity. Tyramide signal amplification (Invitrogen; Carlsbad, CA) was then conducted as described by the manufacturer. In brief, sections were rinsed with PBS and incubated in 10\% normal swine serum (Vector Laboratories; Burlingame, CA) for 1 hour. Sections were then incubated with horseradish peroxidase (HRP)-conjugated sheep anti-DIG Fab fragments (Roche Applied Science) for 1 hour and rinsed with PBS. Oregon Green 488-conjugated tyramide diluted in amplification buffer/0.0015\% $\mathrm{H}_{2} \mathrm{O}_{2}$ was applied to the sections for 10 minutes. Sections were rinsed with PBS and diluted Oregon Green 488-conjugated tyramide was reapplied for 10 minutes. Sections were then incubated with Alexa Fluor 488-conjugated goat anti-fluorescein/Oregon Green IgG (Invitrogen) overnight at $4^{\circ} \mathrm{C}$. To visualize brain endothelial cells, sections were incubated with mouse anti-mouse glucose transporter 1 (GLUT1) IgG (Abcam; Cambridge, MA) overnight at $4^{\circ} \mathrm{C}$. To detect GLUT1, sections were incubated with Alexa Fluor 680-conjugated donkey antimouse IgG (Invitrogen) for 1 hour.

\section{Immunodetection of PDGFR $\beta$, Desmin, Chondroitin Sulfate Proteoglycan NG2 and the $x$ LacZ4 transgenic reporter}

The brains of 6-8 month old 129S1/SvlmJ mice were studied for co-localization of PDGFR $\beta$ and the wellestablished pericyte marker Chondroitin Sulfate Proteoglycan NG2 (NG2) [7,17-19]. Mice were anesthetized and transcardially perfused as described above (see Animals). Snap frozen brain sections were cyrosectioned at $14 \mu \mathrm{m}$ and subsequently fixed in acetone for 10 minutes at $-20^{\circ} \mathrm{C}$. Sections were incubated in $10 \%$ normal swine serum (Vector Laboratories) for 1 hour at room temperature. Sections were then incubated with goat 
anti-mouse PDGFR $\beta$ IgG (R\&D systems; Minneapolis, $\mathrm{MN}$ ) and rabbit anti-rat NG2 IgG (Millipore; Billerica, MA) which cross-reacts with mouse NG2 overnight at $4{ }^{\circ} \mathrm{C}$. For PDGFR $\beta$ and NG2 detection sections were incubated with Alexa Fluor 546-conjugated donkey antigoat IgG (Invitrogen) and Alexa Fluor 488-conjugated donkey anti-rabbit IgG (Invitrogen), respectively, for 1 hour at room temperature. To visualize brain microvascular endothelial cells, sections were incubated with biotin-conjugated Lycopersicon esculentum (tomato) lectin (Vector Laboratories) for 1 hour at room temperature followed by incubation with AMCA-conjugated streptavidin (Vector Laboratories) for 30 minutes at $37^{\circ} \mathrm{C}$.

To confirm colocalization of PDGFR $\beta$ with the $x$ LacZ4 transgenic reporter, a marker of committed and differentiated, non-proliferating pericytes/vascular smooth muscle cells [20], snap frozen 2 month old $x$ LacZ4 transgenic mouse brain sections were cryosectioned at $14 \mu \mathrm{m}$ and subsequently fixed in a 1:1 solution of acetone and methanol for 10 minutes at room temperature. Sections were blocked and incubated with goat anti- mouse PDGFR $\beta$ IgG (R\&D systems) and mouse anti- $\beta$-galactosidase IgG (Cell Signaling; Danvers, MA) as described above. For PDGFR $\beta$ and LacZ detection sections were incubated with Alexa Fluor 488-conjugated donkey anti-goat IgG (Invitrogen; Carlsbad, CA) and Alexa Fluor 546-conjugated donkey anti-mouse IgG (Invitrogen), respectively, as described above. Brain microvascular endothelial cells were visualized with fluorescent lectin staining as described above.

To study PDGFR $\beta$-positive or desmin-positive pericyte coverage, tissue sections were prepared as described above. Sections were then incubated with goat antimouse PDGFR $\beta$ IgG (R\&D systems) or mouse antihuman desmin IgG (Dako USA; Carpinteria, CA) which cross-reacts with mouse desmin overnight at $4^{\circ} \mathrm{C}$. For PDGFR $\beta$ and desmin detection sections were incubated with Alexa Fluor 546-conjugated donkey anti-goat IgG (Invitrogen; Carlsbad, CA) or Alexa Fluor 546-conjugated donkey anti-mouse IgG (Invitrogen), respectively, for 1 hour at room temperature. Brain microvascular endothelial cells were visualized with fluorescent lectin staining as described above.

\section{Immunodetection of neuronal-specific and astrocyte- specific markers}

The brains of 6-8 month old 129S1/SvlmJ littermate controls and 6-8 month old F7 mice were studied for colocalization of PDGFR $\beta$ with neuronal neurofilament$\mathrm{H}$ (SMI-32), neuronal nuclear antigen A60 (NeuN), or astrocyte glial fibrillary acidic protein (GFAP). Mice were anesthetized as described above and transcardially perfused with $10 \mathrm{~mL}$ PBS containing $5 \mathrm{U} / \mathrm{ml}$ heparin followed by $30 \mathrm{~mL} 4 \%$ PFA. Brains were carefully removed from the skull and immersion fixed in 4\% PFA overnight at $4^{\circ} \mathrm{C}$. Forty $\mu \mathrm{m}$ vibratome-sectioned brain sections were incubated in target antigen retrieval solution, $\mathrm{pH} 9$ (Dako USA) for 15 minutes in a $80^{\circ} \mathrm{C}$ water bath and then blocked and incubated with the following primary antibodies as described above: goat anti-mouse PDGFR $\beta$ (R\&D systems), mouse anti-mouse SMI-32 (Abcam), mouse anti-mouse NeuN (Millipore), and mouse anti-mouse GFAP (Cell Signaling). PDGFR $\beta$ was detected as described above. To detect NeuN, SMI-32, and GFAP sections were incubated with Alexa Fluor 488-conjugated donkey anti-mouse IgG for 1 hour at room temperature. Brain microvascular endothelial cells were visualized with fluorescent lectin staining as described above.

\section{Quantification of PDGFR $\beta$-positive and Desmin-positive pericyte coverage of lectin-positive brain microvessels}

All images were acquired by a blinded investigator using a custom-built Zeiss LSM 510 meta confocal laser scanning microscope (see below) using a Zeiss LD LCI PlanApochromat $25 \times / 0.8 \mathrm{Imm}$ Korr DIC water immersion objective. In each mouse, four different $200 \mu \mathrm{m} \times 200$ $\mu \mathrm{m}$ fields were analyzed per brain region in six nonadjacent sections approximately $100 \mu \mathrm{m}$ apart. PDGFR $\beta$ postive or desmin-positive immunofluorescent and lectin-positive fluorescent signals from brain microvessels $<6 \mu \mathrm{m}$ in diameter were individually subjected to threshold processing and the areas occupied by their respective signals were quantified by a blinded investigator using the NIH Image J software Area measurement tool. The percent of PDGFR $\beta$ pericyte coverage of lectin-positive microvessels was then deteremined by normalizing the PDGFR $\beta$-positive signal to the lectin positive-brain microvessel signal. We used Graph-Pad Prism software for statistical calculations. One-way analysis of variance followed by a Tukey posthoc test were used to determine statistically significant differences in PDGFR $\beta$-positive pericyte coverage amongst different brain regions in both $129 \mathrm{~S} 1 / \mathrm{SvlmJ}$ mice and F7 mice. Mean values are reported plus or minus the standard error of the mean (SEM).

\section{Fluorescent in situ PDGF-B/PDGFR $\beta$ proximity ligation assay}

The brains of 6-8 month old 129S1/SvlmJ mice were studied to determine the localization of PDGF-B/ PDGFR $\beta$ interactions in situ. Mice were anesthetized and transcardially perfused as described above (see Animals). Frozen brain sections were cryosectioned at $14 \mu \mathrm{m}$. Sections were rehydrated with PBS and blocked with $10 \%$ normal swine serum (Vector Laboratories) for 1 hour at room temperature. Sections were then incubated with $150 \mathrm{ng} / \mathrm{mL}$ mouse recombinant PDGF-BB 
(GenWay Biotech, Inc.; San Diego, CA) for 1 hour at room temperature. Sections were washed with PBS containing $0.05 \%$ triton $\mathrm{X}-100$ and subsequently fixed with 4\% PFA for 10 minutes at room temperature. Sections were incubated with goat anti-mouse PDGFR $\beta$ IgG (R\&D systems) and rabbit anti-human PDGF-B IgG (Thermo Scientific; Rockford, IL) which cross reacts with mouse PDGF-B overnight at $4^{\circ} \mathrm{C}$. Proximity ligation was then conducted in situ as described by the manufacturer (Olink Bioscience; Uppsala, SE) utilizing the Duolink II PLA probe anti-goat PLUS, Duolink II PLA probe anti-rabbit MINUS, and Duolink II detection reagents orange to visualize PDGF-B/PDGFR $\beta$ interactions. Following serial SSC washes, sections were rinsed with PBS were incubated with biotin-conjugated Lycopersicon esculentum lectin (Vector Laboratories) overnight at $4{ }^{\circ} \mathrm{C}$. To visualize brain microvascular endothelial cells and PDGFR $\beta$, sections were incubated with Dylight 649-conjugated streptavidin (Vector Laboratories) and Alexa Fluor 488-conguated donkey anti-goat IgG (Invitrogen), respectively, for 1 hour at room temperature.

\section{Laser scanning confocal microscopy imaging}

All images were acquired using a custom-built Zeiss LSM 510 meta confocal laser scanning microscope with a Zeiss LD LCI Plan-Apochromat 25×/0.8 Imm Korr DIC, C-Apochromat $40 \mathrm{X}$ or $63 \mathrm{X}$ water immersion objective (Carl Zeiss Microimaging Inc.; Thronwood, $\mathrm{NY}$ ). A $488 \mathrm{~nm}$ argon laser was used to excite Alexa Fluor 488 and the emission was collected through a 500-550 band-pass filter. A $543 \mathrm{HeNe}$ laser was used to excite Alexa Fluor 546 and the emission was collected through a 560-615 band-pass filter. A $633 \mathrm{HeNe}$ laser was used to excite Alexa Fluor 680 and Dylight 649 and the emission was collected through a $650-710$ band-pass filter. An $800 \mathrm{~nm}$ mode-locked femtosecond pulsed DeepSee Ti:sapphire laser (Spectra Physics; Santa Clara, CA) was used for AMCA excitation and emission was collected using 405-450 nm band-pass filter.

\section{Acknowledgements}

We thank Dr. P. Soriano for providing the F7 mice and Dr. M. Tallquist for providing the xLacZ4 mice. This work was supported by R37AG023084 and R37NS34467.

\section{Authors' contributions \\ EAW conducted fluorescent in situ hybridization and proximity ligation experiments and helped to draft the manuscript. RBD conducted all immunostaining experiments, quantified pericyte coverage, and helped to draft the manuscript. BVZ designed and supervised all experiments and wrote the manuscript. All authors read and approved the final manuscript.}

\section{Competing interests}

The authors declare that they have no competing interests.
Received: 22 July 2010 Accepted: 25 August 2010

Published: 25 August 2010

\section{References}

1. Rouget C: Memoire sur le developpement, la structure et les proprieties des capillaires sanguins and lymphatiques. Archs Physiol Norm Pathol 1873, 5:603-633

2. Balabanov R, Dore-Duffy P: Role of the CNC microvascular pericyte in the blood-brain barrier. J Neurosci Res 1998, 53:637-644.

3. ladecola C: Neurovascular regulation in the normal brain and in Alzheimer's disease. Nat Rev Neurosci 2004, 5:347-360.

4. Zlokovic BV: The blood-brain barrier in health and chronic neurodegenerative disorders. Neuron 2008, 57:178-201.

5. Segura I, De Smet F, Hohensinner PJ, Ruiz de Almodovar C, Carmeliet P: The neurovascular link in health and disease: an update. Trends $\mathrm{Mol}$ Med 2009, 15:439-451.

6. Shepro D, Morel NM: Pericyte physiology. FASEB J 1993, 7:1031-1038

7. Armulik A, Abramsson A, Betsholtz C: Endothelial/pericyte interactions. Circ Res 2005, 97:512-523.

8. Leveen P, Pekny M, Gebre-Medhin S, Swolin B, Larsson E, Betsholtz C: Mice deficient for PDGF B show renal, cardiovascular, and hematological abnormalities. Genes Dev 1994, 8:1875-1887.

9. Soriano P: Abnormal kidney development and hematological disorders in PDGF beta-receptor mutant mice. Genes Dev 1994, 8:1888-1896.

10. Lindahl P, Johansson BR, Leveen P, Betsholtz C: Pericyte loss and microaneurysm formation in PDGF-B-deficient mice. Science 1997 277:242-245

11. Lindblom $P$, Gerhardt $H$, Liebner $S$, Abramsson A, Enge $M$, Hellstrom $M$, Backstrom G, Fredriksson S, Landegren U, Nystrom HC, Bergstrom G, Dejana E, Ostman A, Lindahl P, Betsholtz C: Endothelial PDGF-B retention is required for proper investment of pericytes in the microvessel wall. Genes Dev 2003, 17:1835-1840.

12. Tallquist MD, French WJ, Soriano P: Additive effects of PDGF receptor beta signaling pathways in vascular smooth muscle cell development. PLOS Biol 2003, 1:E52

13. Gaengel K, Genove G, Armulik A, Betsholtz C: Endothelial-mural cell signaling in vascular development and angiogenesis. Arterioscler Thromb Vasc Biol 2009, 29:630-638.

14. Hellstrom M, Kalen M, Lindahl P, Abramsson A, Betsholtz C: Role of PDGF-B and PDGFR-beta in recruitment of vascular smooth muscle cells and pericytes during embryonic blood vessel formation in the mouse. Development 1999, 126:3047-3055.

15. Heldin CH, Ostman A, Ronnstrand L: Signal transduction via plateletderived growth factor receptors. Biochim Biophys Acta 1998, 1378:F79-113.

16. Diaz-Flores L, Gutierrez R, Madrid JF, Varela H, Valladares F, Acosta E, MartinVasallo $P$, Diaz-Flores L Jr: Pericytes. Morphofunction, interactions and pathology in a quiescent and activated mesenchymal cell niche. Histol Histopathol 2009, 24:909-969.

17. Peppiatt CM, Howarth C, Mobbs P, Attwell D: Bidirectional control of CNS capillary diameter by pericytes. Nature 2006, 443:700-704.

18. Pfister F, Feng $Y$, vom Hagen F, Hoffmann S, Molema G, Hillebrands JL, Shani M, Deutsch U, Hammes HP: Pericyte migration: a novel mechanism of pericyte loss in experimental diabetic retinopathy. Diabetes 2008, 57:2495-2502.

19. Yemisci M, Gursoy-Ozdemir Y, Vural A, Can A, Topalkara K, Dalkara T: Pericyte contraction induced by oxidative-nitrative stress impairs capillary reflow despite successful opening of an occluded cerebral artery. Nat Med 2009, 15:1031-1037.

20. Tidhar A, Reichenstein M, Cohen D, Faerman A, Copeland NG, Gilbert DJ, Jenkins NA, Shani M: A novel transgenic marker for migrating limb muscle precursors and for vascular smooth muscle cells. Dev Dyn 2001, 220:60-73.

21. Song S, Ewald AJ, Stallcup W, Werb Z, Bergers G: PDGFRbeta+ perivascular progenitor cells in tumours regulate pericyte differentiation and vascular survival. Nat Cell Biol 2005, 7:870-879.

22. Kokovay E, Li L, Cunningham LA: Angiogenic recruitment of pericytes from bone marrow after stroke. J Cereb Blood Flow Metab 2006, 26:545-555.

23. Ohi $Y$, Ishii $Y$, Haji A, Noguchi S, Sasaoka T, Fujimori T, Nabeshima $Y$, Sasahara M, Hattori Y: Platelet-derived growth factor (PDGF)-BB inhibits 
AMPA receptor-mediated synaptic transmission via PDGF receptor-beta in murine nucleus tractus solitarius. Brain Res 2007, 1159:77-85.

24. Ohi Y, Ishii $Y$, Sasahara M, Haji A: Involvement of platelet-derived growth factor-BB and its receptor-beta in hypoxia-induced depression of excitatory synaptic transmission in the nucleus tractus solitarius of mice. J Pharmacol Sci 2010, 112:477-481.

25. Soderberg O, Gullberg M, Jarvius M, Ridderstrale K, Leuchowius K, Jarvius J, Wester K, Hydbring P, Bahram F, Larsson LG, Landegren U: Direct observation of individual endogenous protein complexes in situ by proximity ligation. Nat Methods 2006, 3:995-1000.

26. Soderberg O, Leuchowius KJ, Gullberg M, Jarvius M, Weibrecht I, Larsson $L G$, Landegren $\mathrm{U}$ : Characterizing proteins and their interactions in cells and tissues using the in situ proximity ligation assay. Methods 2008, 45:227-232.

27. Cahoy JD, Emery B, Kaushal A, Foo LC, Zamanian JL, Christopherson KS, Xing Y, Lubischer JL, Krieg PA, Krupenko SA, Thompson WJ, Barres BA: A transcriptome database for astrocytes, neurons, and oligodendrocytes: a new resource for understanding brain development and function. $J$ Neurosci 2008, 28:264-267.

28. Cahoy CNS Celltypes Exonarray. [http://innateimmunity.mit.edu/cgi-bin/ exonarraywebsite/plot_selector_cahoy.pl?exact $=1 \&$ gene=Pdgfrb].

29. Zheng L, Ishii Y, Tokunaga A, Hamashima T, Shen J, Zhao QL, Ishizawa S, Fujimori T, Nabeshima Y, Mori H, Kondo T, Sasahara M: Neuroprotective effects of PDGF against oxidative stress and the signaling pathway involved. J Neurosci Res 2010, 88:1273-1284.

30. Tosh D, Slack JM: How cells change their phenotype. Nat Rev Mol Cell Biol 2002, 3:187-194.

31. Hsieh J, Gage FH: Epigenetic control of neural stem cell fate. Curr Opin Genet Dev 2004, 14:461-469.

32. Liu XS, Zhang ZG, Zhang RL, Gregg SR, Meng H, Chopp M: Comparison of in vivo and in vitro gene expression profiles in subventricular zone neural progenitor cells from the adult mouse after middle cerebral artery occlusion. Neuroscience 2007, 146:1053-1061.

33. Ishii Y, Oya T, Zheng L, Gao Z, Kawaguchi M, Sabit H, Matsushima T, Tokunaga A, Ishizawa S, Hori E, Nabeshima Y, Sasaoka T, Fujimori T, Mori H, Sasahara M: Mouse brains deficient in neuronal PDGF receptor-beta develop normally but are vulnerable to injury. J Neurochem 2006, 98:588-600.

34. Dore-Duffy P, Katychev A, Wang X, Van Buren E: CNS microvascular pericytes exhibit multipotential stem cell activity. I Cereb Blood Flow Metab 2006, 26:613-624.

35. Heldin CH, Backstrom G, Ostman A, Hammacher A, Ronnstrand L, Rubin K, Nister M, Westermark B: Binding of different dimeric forms of PDGF to human fibroblasts: evidence for two separate receptor types. EMBO $J$ 1988, 7:1387-1393

36. Claesson-Welsh L, Eriksson A, Westermark B, Heldin CH: CDNA cloning and expression of the human A-type platelet-derived growth factor (PDGF) receptor establishes structural similarity to the B-type PDGF receptor. Proc Natl Acad Sci USA 1989, 86:4917-4921.

37. Eriksson A, Siegbahn A, Westermark B, Heldin CH, Claesson-Welsh L: PDGF alpha- and beta-receptors activate unique and common signal transduction pathways. EMBO J 1992, 11:543-550.

38. Vignais L, Oumesmar BN, Baron-Van Evercooren AB: PDGF-alpha receptor is expressed by mature neurones of the central nervous system. Neuroreport 1995, 6:1993-1996.

39. Oumesmar BN, Vignais L, Baron-Van Evercooren A: Developmental expression of platelet-derived growth factor alpha-receptor in neurons and glial cells of the mouse CNS. J Neurosci 1997, 17:125-139.

40. Tabrizi P, Wang L, Seeds N, McComb JG, Yamada S, Griffin JH, Carmeliet P, Weiss MH, Zlokovic BV: Tissue plasminogen activator (tPA) deficiency exacerbates cerebrovascular fibrin deposition and brain injury in a murine stroke model: studies in TPA-deficient mice and wild-type mice on a matched genetic control. Aterioscler Thromb Vasc Biol 1999, 19:2801-2806.

41. Connolly ES Jr, Winfree CJ, Stern DM, Solomon RA, Pinsky DJ: Procedural and strain-related variables significantly affect outcome in a murine model of focal cerebral ischemia. Neurosurgery 1996, 38:523-531, discussion 532.

42. Mutant mice and neuroscience: recommendations concerning genetic background. Banbury Conference on genetic background in mice. Neuron 1997, 19:755-759.
43. Sandberg R, Yasuda R, Pankratz DG, Carter TA, Del Rio JA, Wodicka L, Mayford M, Lockhart DJ, Barlow C: Regional and strain-specific gene expression mapping in the adult mouse brain. Proc Natl Acad Sci USA 2000, 97:11038-11043.

44. Smits A, Kato M, Westermark B, Nister M, Heldin CH, Funa K, Neurotrophic activity of platlet-derived growth factor (PDGF): Rat neuronal cells possess functional PDGF beta-type receptors and respond to PDGF. Proc Natl Acad Sci USA 1991, 88:8159-8163.

45. Berger M, Bergers G, Arnold B, Hammerling GJ, Ganss R: Regulator of Gprotein signaling-5 induction in pericytes coincides with active vessel remodeling during neovascularization. Blood 2005, 105:1094-1101.

46. Thompson CL, Pathak SD, Jeromin A, Ng LL, MacPherson CR, Mortrud MT, Cusick A, Riley ZL, Sunkin SM, Bernard A, Puchalski RB, Gage FH, Jones AR, Bajic VB, Hawrylycz MJ, Lein ES: Genomic anatomy of the hippocampus. Neuron 2008, 60:1010-1021.

doi:10.1186/1750-1326-5-32

Cite this article as: Winkler et al:: Pericyte-specific expression of PDGF beta receptor in mouse models with normal and deficient PDGF beta receptor signaling. Molecular Neurodegeneration 2010 5:32.

\section{Submit your next manuscript to BioMed Central and take full advantage of:}

- Convenient online submission

- Thorough peer review

- No space constraints or color figure charges

- Immediate publication on acceptance

- Inclusion in PubMed, CAS, Scopus and Google Scholar

- Research which is freely available for redistribution

Submit your manuscript at www.biomedcentral.com/submit
Ciomed Central 